\title{
Numerical simulations of the light propagation in the gravitational field of moving bodies
}

\begin{abstract}
S. A. Klioner and M. Peip
Lohrmann Observatory, Dresden Technical University, Mommsenstr. 13, 01062 Dresden, Germany

Received 14 May 2003 / Accepted 1 August 2003

Abstract. One of the most subtle points in the modern relativistic models for microarcsecond astrometrical observations is the treatment of the influence of translational motion of gravitating bodies on the light propagation. This paper describes numerical simulations of the light propagation in the gravitational field of moving gravitating bodies and summarizes the underlying theory. The simulations include high-precision numerical integrations of both post-Newtonian and post-Minkowskian differential equations of light propagation and a detailed comparison of the results of the numerical integrations with various available approximate analytical formulas. The simulations has been performed both for hypothetical bodies with various parameters of trajectories as well as for all the major bodies of the solar system using the JPL ephemeris DE405/LE405 to calculate their motion.

It is shown that for an accuracy of $\sim 0.2 \mu$ as it is sufficient to use the well-known solution for the light propagation in the field of a motionless mass monopole and substitute in that solution the position of the body at the moment of closest approach between the actual trajectory of the body and the unperturbed light path (as was first suggested by Hellings 1986). For a higher accuracy one should use either the post-Newtonian solution for uniformly moving bodies (Klioner \& Kopeikin 1992) or the post-Minkowskian solution for arbitrarily moving bodies (Kopeikin \& Schäfer 1999). For astrometric observations performed from within the solar system these two solutions guarantee the accuracy of $\sim 0.002 \mu$ as and are virtually indistinguishable from each other.
\end{abstract}

Key words. astrometry - reference systems - relativity - gravitational lensing

\section{Introduction}

It is widely known that extremely high accuracy of the future space-born astrometric missions like GAIA (ESA 2000; Perryman et al. 2001; Bienayme \& Turon 2002) and SIM (Shao 1998) makes it necessary to formulate the reduction model of positional observations in a form fully consistent with General Relativity Theory (GRT). The relativistic models of positional observations has been formulated by several groups of authors: Klioner (1989), Brumberg et al. (1990), Klioner \& Kopeikin (1992), de Felice et al. (1998, 2000, 2001), Klioner (2003a). This paper is devoted to an investigation of one subtle point in any microarcsecond relativistic model of positional observations. Namely, the influence of the translational motion of gravitating bodies on the light propagation is investigated here in great detail.

After the pioneering work of Hellings (1986) where it was suggested to compute the positions of the gravitating bodies at the moment of closest approach between the body and the unperturbed light ray and substitute these positions into the wellknown solution for light propagation in the gravitational field of a system of motionless bodies, several authors have

Send offprint requests to: S. A. Klioner, e-mail: klioner@rcs.urz.tu-dresden.de succeeded in formulating more rigorous approaches to the problem (Klioner 1989, 1991; Klioner \& Kopeikin 1992; Kopeikin \& Schäfer 1999; Kopeikin \& Mashhoon 2002). Detailed historical overviews can be found in Introduction of Kopeikin \& Schäfer (1999) and in Sect. 6 of Klioner (2003a) (see also Klioner 2003b).

In this paper we perform extensive numerical simulations aimed at clarifying the ability of various approximate analytical formulas to reproduce the gravitational light deflection in the field of the solar system at the level of $0.1-1 \mu$ as as required by GAIA and SIM.

Possible ways to compute the light trajectory in the gravitational field of moving bodies are summarized in Sect. 2. Section 3 explains the general layout of the simulations. The results of the simulations are discussed in Sect. 4. The suggestions for practical relativistic modeling of high-accuracy positional observations depending on the goal accuracy are formulated in Sect. 5. In the Appendices we summarize the theoretical formulas concerning the influence of the translational motion of the gravitating bodies on light propagation. Most of these formulas are used in the simulations. Appendix A contains general formulas for null geodesics in the weak-field approximation. Both post-Newtonian and post-Minkowskian equations are given there. The most important theoretical 
results for light propagation in the post-Newtonian approximation are given in Appendix B. The equations of light propagation in the post-Minkowskian approximation are discussed in Appendix C. The two point boundary problem for analytical solutions is discussed in Appendix D.

\section{Possible ways to calculate the light propagation in the field of moving bodies}

According to the theory described in the Appendices there are several ways to calculate the light trajectory in the gravitational field of moving mass monopoles:

1. Numerical integration of the post-Minkowskian differential equations of light propagation (C.11)-(C.22) with initial conditions (C.25) - (C.27).

2. Analytical post-Minkowskian solution (C.28)-(C.33) for arbitrarily moving bodies (the solution for the position of the photon (C.28) contains an integral that can be computed numerically, or estimated to be negligible for a particular purpose and thus neglected).

3. Numerical integration of the post-Newtonian equations of light propagation (B.5)-(B.12) with initial conditions (B.13)-(B.14).

4. Analytical post-Newtonian solution for uniformly moving mass monopoles (B.15)-(B.26) with two free parameters $\boldsymbol{x}_{\mathrm{A} 0}$ and $\boldsymbol{v}_{\mathrm{A}}$ to be related to the actual trajectory of the body.

As discussed in the Appendices below, the post-Minkowskian approximation scheme deals with expansions in powers of the gravitational constant $G$. Velocities of gravitating bodies are not considered as small in the post-Minkowskian approximation scheme which is sometimes called the weak-field fastmotion approximation. The first post-Minkowskian approximation implies that all terms of order $O\left(G^{2}\right)$ are neglected. The post-Newtonian approximation scheme operates with expansions in powers of $c^{-1}$. In the post-Newtonian approximation scheme velocities of gravitating bodies are considered to be small. This approximation scheme is sometimes called the weak-field slow-motion approximation. In the first postNewtonian approximation, terms of order $O\left(c^{-4}\right)$ are neglected in the equations of light propagation. One can prove that in the case of light propagation the formulas of the first postNewtonian approximation are linear in $G$ and, therefore, contained in those of the first post-Minkowskian approximation (see e.g. Appendix C).

The most accurate way to calculate the light propagation is clearly the first one, i.e. numerical integration of the postMinkowskian differential equations of motion for the photon. The errors of those differential equations of motion come from the effects of the second post-Minkowskian (or postNewtonian) approximation which are known to be negligible for the solar system applications. Numerical integration of the post-Newtonian equations of motion for the photon (B.5)(B.12) can also be used to calculate the light trajectory in the field of arbitrarily moving bodies. However, since the postNewtonian equations of motion are contained in the postMinkowskian equations of motion (Appendix C), the former can be used for an internal consistency check of the whole calculation rather than for an independent computation of the light path. Our numerical experiments show that for solar system applications the results of numerical integrations of the post-Newtonian and post-Minkowskian equations of motion are identical within the errors of the first post-Minkowskian approximation.

The analytical post-Minkowskian solution given in Appendix C.4 is surely the most accurate analytical solution for the problem. However, (1) the solution for the photon's position involves an integral which should be in principle computed numerically, and (2) the post-Minkowskian solution is relatively expensive as far as the computing time is concerned since it contains the retarded moment of time to be computed by iterations (see below). On the other hand, the full accuracy of the analytical post-Minkowskian solution is not necessary to attain the accuracy of $1 \mu$ as for the solar system applications. Simpler analytical solutions of sufficient accuracy can be found instead.

The fully analytical post-Newtonian solution given in Appendix B.4 describes the light trajectory in the field of uniformly moving gravitating bodies having the coordinates

$\boldsymbol{x}_{\mathrm{A}}(t)=\boldsymbol{x}_{\mathrm{A} 0}+\boldsymbol{v}_{\mathrm{A}}\left(t-t_{0}\right)$,

where $\boldsymbol{x}_{\mathrm{A} 0}$ and $\boldsymbol{v}_{\mathrm{A}}$ are two arbitrary constant vectors. These constants can be related to the actual trajectory of the body $x_{\mathrm{A}}^{\mathrm{eph}}(t)$ in different ways in the hope that the errors related to the nonuniformity of the body's trajectory will be minimized in some sense. The principal goal of this paper is to check if the analytical post-Newtonian solution with some reasonable choice of the constants $\boldsymbol{x}_{\mathrm{A} 0}$ and $\boldsymbol{v}_{\mathrm{A}}$ can describe the gravitational light deflection with an accuracy of $0.1-1 \mu$ as.

For the analytical post-Newtonian solution one has to choose either a fixed point or a straight line as the model trajectory $\boldsymbol{x}_{\mathrm{A}}(t)$ of the body which should be distinguished from the actual trajectory of the body $\boldsymbol{x}_{\mathrm{A}}^{\mathrm{eph}}(t)$. In this paper we consider six choices for the constants $\boldsymbol{x}_{\mathrm{A} 0}$ and $\boldsymbol{v}_{\mathrm{A}}$ in the post-Newtonian solution: four solutions for a body at rest named $P_{1}, P_{2}, P_{3}$ and $P_{3}^{\prime}$ and two solutions for a body moving with constant velocity $L_{1}$ and $L_{2}$ (see Fig. 1). Each of the considered solutions uses the actual position (and possibly velocity) of the body at some reference moment of time $t=t_{\text {ref }}$. The most simple solution $P_{1}$ uses the fixed position $x_{\mathrm{A}}^{\text {eph }}\left(t_{\mathrm{o}}\right)$ of the body at the moment of observation $t_{\mathrm{ref}}=t_{\mathrm{o}}$. The fixed position $\boldsymbol{x}_{\mathrm{A}}^{\mathrm{eph}}\left(t^{\mathrm{ca}}\right)$ of the body at the moment $t_{\text {ref }}=t^{\text {ca }}$ of closest approach between the body and the unperturbed light ray is used for the second solution $P_{2}$. The moment $t^{\text {ca }}$ can be calculated as

$$
\begin{aligned}
& t^{\mathrm{ca}}=\max \left(t_{\mathrm{e}}, t_{\mathrm{o}}-\max \left(0, \frac{\boldsymbol{g} \cdot\left(\boldsymbol{x}_{\mathrm{p}}\left(t_{\mathrm{o}}\right)-\boldsymbol{x}_{\mathrm{A}}^{\mathrm{eph}}\left(t_{\mathrm{o}}\right)\right)}{c|\boldsymbol{g}|^{2}}\right)\right), \\
& \boldsymbol{g}=\boldsymbol{\mu}-\frac{1}{c} \dot{\boldsymbol{x}}_{\mathrm{A}}^{\mathrm{eph}}\left(t_{\mathrm{o}}\right),
\end{aligned}
$$

where $t_{\mathrm{e}}$ is the moment of emission of the photon, and $\boldsymbol{x}_{\mathrm{p}}(t)$ is the (unperturbed) trajectory of the photon. If the source is situated outside of the solar system one can put $t_{\mathrm{e}}=-\infty$ and the outer "max" can be omitted in (2). The retarded moment of 


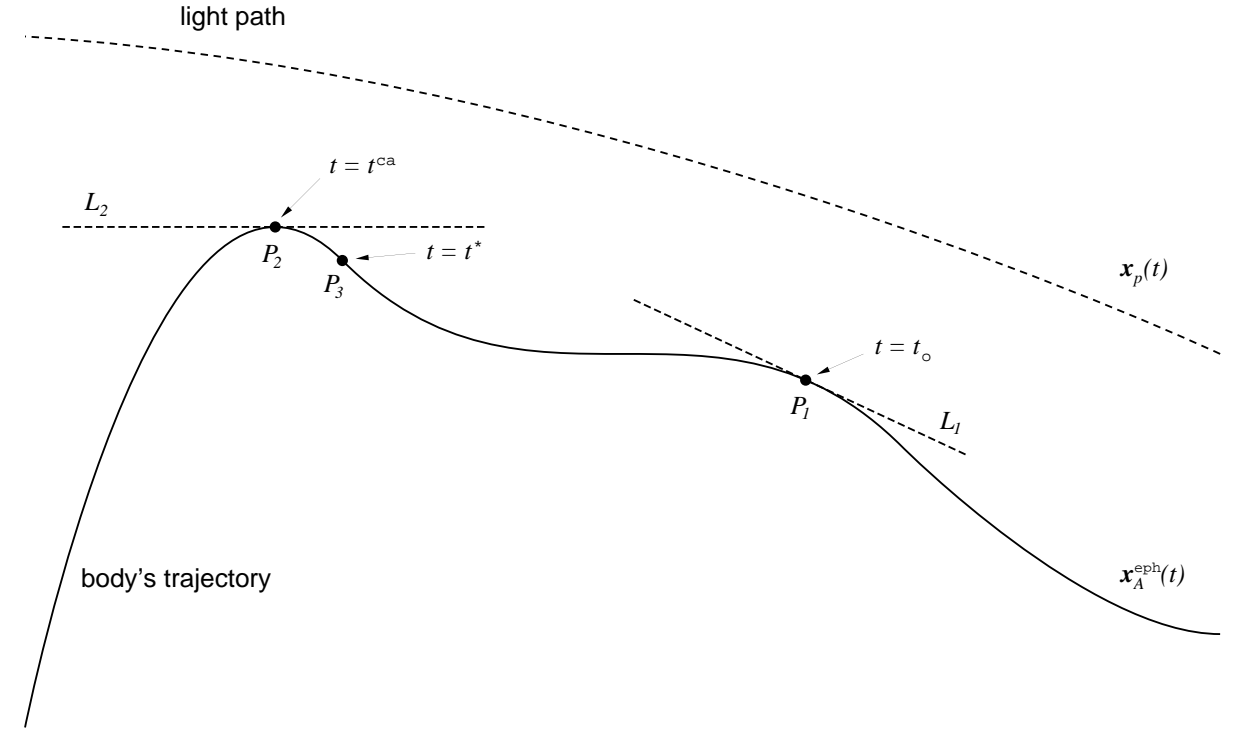

Fig. 1. Actual trajectory $x_{\mathrm{A}}^{\mathrm{eph}}(t)$ of body A and the five simplified trajectories used to model the light propagation within the post-Newtonian approximation scheme: three fixed positions $P_{1}\left(x_{\mathrm{A}}(t)=x_{\mathrm{A}}^{\mathrm{eph}}\left(t_{\mathrm{o}}\right)=\right.$ const $), P_{2}\left(x_{\mathrm{A}}(t)=x_{\mathrm{A}}^{\mathrm{eph}}\left(t^{\mathrm{ca}}\right)=\right.$ const $)$ and $P_{3}\left(x_{\mathrm{A}}(t)=\boldsymbol{x}_{\mathrm{A}}^{\mathrm{eph}}\left(t^{*}\right)=\right.$ const $)$, and two trajectories with constant velocity $L_{1}$ $\left(x_{\mathrm{A}}(t)=\boldsymbol{x}_{\mathrm{A}}^{\text {eph }}\left(t_{\mathrm{o}}\right)+\dot{\boldsymbol{x}}_{\mathrm{A}}^{\text {eph }}\left(t_{\mathrm{o}}\right)\left(t-t_{\mathrm{o}}\right)\right)$ and $L_{2}$ $\left(x_{\mathrm{A}}(t)=x_{\mathrm{A}}^{\mathrm{eph}}\left(t^{\mathrm{ca}}\right)+\dot{x}_{\mathrm{A}}^{\mathrm{eph}}\left(t^{\mathrm{ca}}\right)\left(t-t^{\mathrm{ca}}\right)\right)$. The position $P_{3}^{\prime}$ mentioned in the text is not shown on the sketch. It is situated on the actual trajectory of the body close to $P_{3}$.
Table 1. Choice of the constants for the six analytical post-Newtonian solutions for the light trajectory considered in the present paper (see text for further explanations).

\begin{tabular}{llll}
\hline \hline solution & $t_{\text {ref }}$ & $\boldsymbol{x}_{\mathrm{A} 0}$ & $\boldsymbol{v}_{\mathrm{A}}$ \\
\hline$P_{1}$ & $t_{\mathrm{o}}$ & $\boldsymbol{x}_{\mathrm{A}}^{\mathrm{eph}}\left(t_{\mathrm{o}}\right)$ & 0 \\
$P_{2}$ & $t^{\mathrm{ca}}$ & $\boldsymbol{x}_{\mathrm{A}}^{\mathrm{eph}}\left(t^{\mathrm{ca}}\right)$ & 0 \\
$P_{3}$ & $t^{*}$ & $\boldsymbol{x}_{\mathrm{A}}^{\mathrm{eph}}\left(t^{*}\right)$ & 0 \\
$P_{3}^{\prime}$ & $t^{* \prime}$ & $\boldsymbol{x}_{\mathrm{A}}^{\mathrm{eph}}\left(t^{* \prime}\right)$ & 0 \\
& & & \\
$L_{1}$ & $t_{\mathrm{o}}$ & $\boldsymbol{x}_{\mathrm{A}}^{\mathrm{eph}}\left(t_{\mathrm{o}}\right)$ & $\dot{\boldsymbol{x}}_{\mathrm{A}}^{\mathrm{eph}}\left(t_{\mathrm{o}}\right)$ \\
$L_{2}$ & $t^{\mathrm{ca}}$ & $\boldsymbol{x}_{\mathrm{A}}^{\mathrm{eph}}\left(t^{\mathrm{ca}}\right)$ & $\dot{\boldsymbol{x}}_{\mathrm{A}}^{\mathrm{eph}}\left(t^{\mathrm{ca}}\right)$ \\
\hline
\end{tabular}

time $t^{*}$ is used as $t_{\text {ref }}$ in the solution $P_{3}$. This moment of time is defined by

$t^{*}+\frac{1}{c}\left|\boldsymbol{x}_{\mathrm{p}}(t)-\boldsymbol{x}_{\mathrm{A}}^{\mathrm{eph}}\left(t^{*}\right)\right|=t$.

The moment $t^{*}$ is relatively expensive to calculate since the Eq. (4) is an implicit one and one has to use some kind of iteration to solve it (e.g., Newton's method). That is why one can try to substitute $t^{*}$ by its simplified version $t^{* \prime}$ which can be directly calculated

$t^{* \prime}=t-\frac{1}{c}\left|x_{\mathrm{p}}(t)-x_{\mathrm{A}}^{\mathrm{eph}}(t)\right|$.

The solution $P_{3}^{\prime}$ uses the position $x_{\mathrm{A}}^{\mathrm{eph}}\left(t^{* \prime}\right)$ of the body at $t_{\text {ref }}=t^{* \prime}$.

The solutions $L_{1}$ and $L_{2}$ are the solutions for a body moving with a constant velocity. In these two cases the parameters $\boldsymbol{x}_{\mathrm{A} 0}$ and $v_{\mathrm{A}}$ of (B.15)-(B.26) are chosen to coincide with the actual position and velocity of the body at the moments $t_{\mathrm{ref}}=t_{\mathrm{o}}$ and $t_{\text {ref }}=t^{\mathrm{ca}}$, respectively (therefore, the trajectories of the bodies used in $L_{1}$ and $L_{2}$ are tangents to the actual trajectory of the body at these two moments of time). The choice of the trajectories $\boldsymbol{x}_{\mathrm{A}}(t)$ are summarized in Table 1 and Fig. 1.

\section{Simulations}

It is clear that numerical integration of the differential equations of light propagation can be performed only for sources situated at some finite distance from the gravitating body (the end point of numerical integrations is anyway at some finite distance since it is defined by the position of the observer). Light propagation from a source at a finite distance to the observer represents a two point boundary problem for the differential equations of light propagation. As discussed by Klioner (2003a) the goal of the relativistic reduction of observations in this case is to relate the unit direction $\boldsymbol{n}$ of the light propagation at the moment of observation to the unit direction $\boldsymbol{k}$ from the point of light emission $\boldsymbol{x}_{\mathrm{p}}\left(t_{0}\right)$ to the point of light observation $\boldsymbol{x}_{\mathrm{p}}(t)$ (see, Fig. 2).

In each individual simulation we fix the points of emission and observation for all the solutions. These points are computed by numerical integration of the post-Minkowskian differential equations, so that for this most accurate solution we compute vectors $\boldsymbol{n}, \boldsymbol{\mu}, \boldsymbol{x}_{\mathrm{p}}\left(t_{0}\right), \boldsymbol{x}_{\mathrm{p}}(t)$ and $\boldsymbol{k}$ with the maximal possible accuracy. Using the formulas given in Appendix D we then solve the two point boundary problem for all the analytical solutions discussed in our simulations and compare the vector $\boldsymbol{n}$ from the post-Minkowskian numerical integration to the vectors $\boldsymbol{n}$ from the analytical solutions. We choose the distance between the point of emission and the gravitating body sufficiently large so that the differences between the vectors $n$ do not change (within an accuracy of $0.001 \mu$ as) when we further increase the distance. Therefore, we can claim that our results are valid also for sources at infinity. Moreover, the simulations have shown that the differences between vectors $\boldsymbol{n}$ for the points of emission at lower distances are smaller than those for sources at infinity. This means that the maximal errors given below are valid also for sources situated at finite distances from the observer (e.g. for the solar system objects).

In order to test the analytical formulas in different situations and check internal consistency of the simulations we have done three independent series of simulations with different choices for the trajectories $\boldsymbol{x}_{\mathrm{A}}^{\mathrm{eph}}(t)$ of the bodies. For all the simulations 


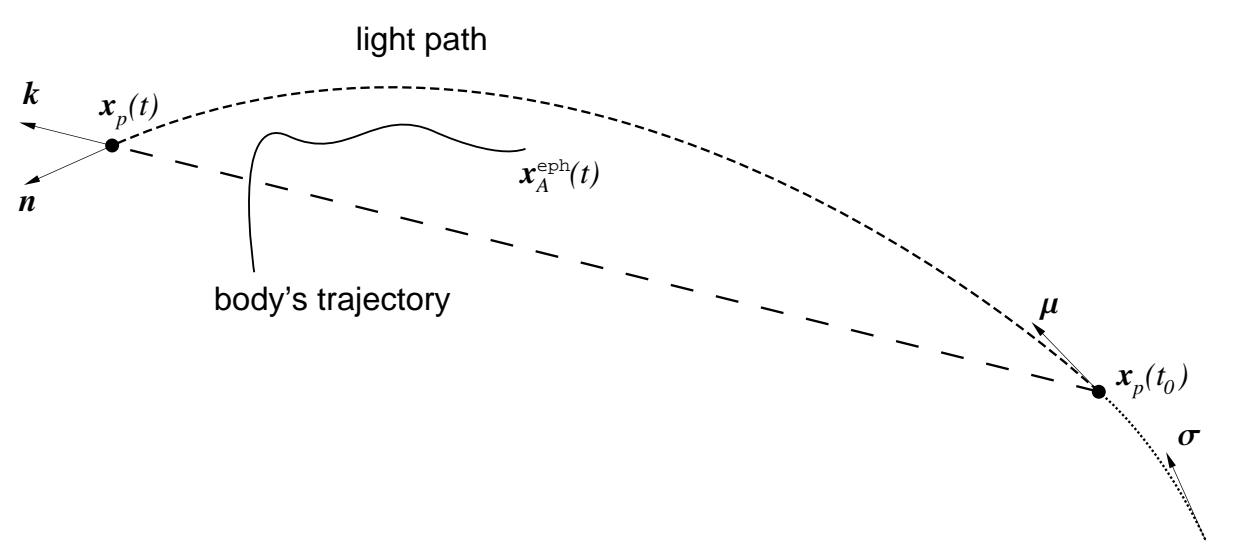

Fig. 2. Four vectors appearing in the calculations: vector $\boldsymbol{\mu}$ is the unit light direction at the point of emission $\boldsymbol{x}_{\mathrm{p}}\left(t_{0}\right), \boldsymbol{n}$ is the unit light direction at the point of observation $\boldsymbol{x}_{\mathrm{p}}(t), \boldsymbol{k}$ is the unit coordinate direction from $\boldsymbol{x}_{\mathrm{p}}\left(t_{0}\right)$ to $\boldsymbol{x}_{\mathrm{p}}(t)$, and $\boldsymbol{\sigma}$ is the unit direction of the light propagation for $t \rightarrow-\infty$. Formal definitions of these vectors are given in Appendix D. the masses of the gravitating bodies have been taken from the JPL ephemeris DE405/LE405 while the radii and other parameters of the bodies were taken from Weissman et al. (1999). The three series of simulations can be described as follows.

A. Parabolic trajectories with constant acceleration (Table 2). For each trajectory the velocity and the acceleration of the body at the moment of closest approach between the body and the photon coincide with the maximal possible barycentric velocity and acceleration of the corresponding body of the solar system. The impact parameter for each trajectory is chosen so that at the moment of closest approach the distance between the photon and the body is equal to the radius of the corresponding body (expect for the three lines in Table 2 with fixed minimal allowed angular distance $\psi_{\text {min }}$ between the gravitating body and the direction of light propagation as seen by the observer; the minimal avoidance angle for each of these three bodies (Earth, Sun and Moon) are calculated from the condition that the minimal Sun avoidance angle is $\psi_{\min }^{\odot}=35^{\circ}$ ). The distance between the observer (satellite) and the gravitating body is taken to be the maximal possible distance between the GAIA satellite and the corresponding body of the solar system (some of the effects under study become larger with increasing this distance and it is important to use the maximal possible distance for the simulations). For the calculation of that maximal distance the satellite was supposed to be situated exactly at the Lagrange point $L_{2}$ of the Earth-Sun system. After fixing all these parameters the initial conditions of the photon trajectory are still not unique and this freedom can be used to check all possible mutual orientations of the velocity vector of the photon and the velocity and acceleration vectors of the body at the moment of closest approach. The directions of all these three vectors are independent of each other. Routinely, in a coordinate system where the direction of the velocity of the photon is fixed we check 50 uniformly distributed directions for each of the two other vectors to find the maximal value of the effects under investigation. In several cases we have checked that a finer grid of mutual orientations does not lead to any changes in the maximal differences between the models given in Table 2 .

B. Circular coplanar trajectories (Table 3). The observer is supposed to be situated exactly at the Lagrange point $L_{2}$ of the Earth-Sun system. Each gravitating body moves along a circular orbit with realistic semi-major axis and mean motion. All the orbits are coplanar. All possible configurations of the observer and the gravitating body have been checked with a step of 0.01 of the siderial period of the corresponding body. The impact parameter of the light ray (i.e. the minimal distance between the photon and the body) is chosen to be not smaller than the radius of the body, but can be larger to meet the requirement imposed by the minimal Sun avoidance angle $\psi_{\text {min }}^{\odot}=35^{\circ}$. For all the bodies for which the minimal Sun avoidance angle influences their observability (Sun, Mercury, Venus, Earth and Moon), the simulations has been done with and without taking the minimal Sun avoidance angle of $\psi_{\text {min }}^{\odot}=35^{\circ}$ into account. For each mutual configuration of the body and the observer 36 different initial positions and velocities of the photon have been chosen so that in the view plane of the observer the observed directions of light are uniformly distributed around the observed position of the gravitating body. Table 3 contains maximal differences between the models for all the light trajectories investigated for each of the body.

C. Realistic trajectories on the basis of the JPL ephemeris DE405 (Table 4). Simulations similar to $\mathbf{B}$ have been performed using the JPL numerical ephemeris DE405/LE405 for the trajectories of the gravitating bodies. The orbit of the observer is taken to be a realistic Lissajoustype orbit about the $L_{2}$ point of the Earth-Sun system and was computed using the algorithm suggested by Mignard (2002). For each body the minimal Sun avoidance angle of $35^{\circ}$ has been taken into account while choosing the parameters of the light rays. All mutual configurations of the observer and the gravitating bodies between 1 January 2008 and 31 December 2020 have been checked with a step of 1 day (the total time span covers 4749 days). The impact parameters of the light rays are taken in the same way as for simulation $\mathbf{B}$. Also in the same way as in simulation $\mathbf{B}$ we have checked 36 different directions of the light ray uniformly distributed relative to the line connecting the observer and the gravitating body. We also checked in several cases that increasing the number of the observed light directions from 36 to, say, 360, does not change the maximal differences between the models given in Table 3 .

For simulations A, B and $\mathbf{C}$ an ANSI C program has been written. Since the effects we are looking for can be as small 
Table 2. The result of simulations using parabolic trajectories $\boldsymbol{x}_{\mathrm{A}}^{\mathrm{eph}}(t)$. All quantities are given in $\mu$ as. The second column $\delta$ is the maximal light deflection angle for the body computed with the post-Minkowskian differential equations of light propagation, while $\delta^{2}$ is the approximate value of the post-post-Minkowskian terms which are neglected in our considerations. The maximal errors of the post-Minkowskian analytical solution $(p M)$ and the six post-Newtonian solutions $(p N)$ are given. The meaning of $P_{i}$ and $L_{i}$ is discussed in Sect. 2 and summarized in Table 1. The number " 0.0 " means that the actual value of the effect is less than $0.001 \mu$ as. The bodies designated with a star, e.g. "Mercury", cannot be observed by GAIA because of the minimal Sun avoidance angle of at least $35^{\circ}$. The minimal impact angle $\psi_{\min }$ for the Sun and the Earth coincide with the minimal Sun avoidance angle of $\psi_{\min }^{\odot}=35^{\circ}$, while that for the Moon is approximately calculated from simplified orbits of GAIA and the Moon.

\begin{tabular}{|c|c|c|c|c|c|c|c|c|c|}
\hline body & $\overline{c \delta}$ & 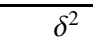 & $\overline{p M}$ & $\overline{p N, P_{1}}$ & $\overline{p N, P_{2}}$ & $\overline{p p N, P_{3}}$ & $\overline{p N, P_{3}^{\prime}}$ & $\overline{p N, L_{1}}$ & $\overline{p N, L_{2}}$ \\
\hline Sun* & $1.76 \times 10^{6}$ & 15.0 & 17.7 & 43.3 & 17.8 & 17.8 & 17.8 & 17.7 & 17.7 \\
\hline $\operatorname{Sun}, \psi_{\min }^{\odot}=35^{\circ}$ & 13600 & 0.001 & 0.001 & 0.003 & 0.002 & 0.002 & 0.002 & 0.001 & 0.001 \\
\hline Mercury* & 82.9 & 0.0 & 0.0 & 93.2 & 0.016 & 0.016 & 0.154 & 0.601 & 0.0 \\
\hline Venus & 493 & 0.0 & 0.0 & 812 & 0.058 & 0.058 & 0.178 & 0.357 & 0.0 \\
\hline Earth* & 574 & 0.0 & 0.0 & 8.08 & 0.058 & 0.058 & 0.058 & 0.0 & 0.0 \\
\hline Earth, $\psi_{\min }=35^{\circ}$ & 3.64 & 0.0 & 0.0 & 0.001 & 0.001 & 0.001 & 0.001 & 0.0 & 0.0 \\
\hline Moon* & 25.9 & 0.0 & 0.0 & 1.44 & 0.003 & 0.003 & 0.003 & 0.0 & 0.0 \\
\hline Moon, $\psi_{\min }=19^{\circ}$ & 0.086 & 0.0 & 0.0 & 0.0 & 0.0 & 0.0 & 0.0 & 0.0 & 0.0 \\
\hline Mars & 116 & 0.0 & 0.0 & 143 & 0.010 & 0.010 & 0.058 & 0.096 & 0.0 \\
\hline Jupiter & 16300 & 0.001 & 0.002 & 26900 & 0.746 & 0.746 & 0.847 & 0.292 & 0.002 \\
\hline Saturn & 5780 & 0.0 & 0.0 & 86100 & 0.196 & 0.196 & 0.250 & 0.036 & 0.0 \\
\hline Uranus & 2530 & 0.0 & 0.0 & 5960 & 0.047 & 0.047 & 0.110 & 0.085 & 0.0 \\
\hline Neptune & 2080 & 0.0 & 0.0 & 6530 & 0.050 & 0.050 & 0.106 & 0.081 & 0.0 \\
\hline
\end{tabular}

Table 3. The maximal difference between the models in the simulations with circular coplanar trajectories $\boldsymbol{x}_{\mathrm{A}}^{\mathrm{eph}}(t)$ for the gravitating bodies. All quantities are given in $\mu$ as. See caption of Table 2 and Sect. 3 for further details.

\begin{tabular}{lrrrrrrrrr}
\hline \hline body & $\delta$ & $\delta^{2}$ & $p M$ & $p N, P_{1}$ & $p N, P_{2}$ & $p N, P_{3}$ & $p N, P_{3}^{\prime}$ & $p N, L_{1}$ & $p N, L_{2}$ \\
\hline Sun* & $1.75 \times 10^{6}$ & 14.8 & 17.5 & 33.3 & 17.5 & 17.5 & 17.5 & 17.5 & 17.5 \\
${\text { Sun, } \psi_{\min }}^{\circ}=35^{\circ}$ & 12900 & 0.001 & 0.001 & 0.002 & 0.001 & 0.001 & 0.001 & 0.001 & 0.001 \\
Mercury $^{*}$ & 83.1 & 0.0 & 0.0 & 315 & 0.013 & 0.013 & 0.091 & 0.192 & 0.0 \\
Mercury, $\psi_{\min }^{\odot}=35^{\circ}$ & 0.007 & 0.0 & 0.0 & 0.0 & 0.0 & 0.0 & 0.0 & 0.0 & 0.0 \\
Venus $^{*}$ & 493 & 0.0 & 0.0 & 33000 & 0.058 & 0.058 & 0.156 & 0.156 & 0.0 \\
Venus, $_{\text {min }}^{\odot}=35^{\circ}$ & 493 & 0.0 & 0.0 & 33300 & 0.058 & 0.058 & 0.145 & 0.144 & 0.0 \\
Earth & 574 & 0.0 & 0.0 & 13.8 & 0.0 & 0.0 & 0.0 & 0.0 & 0.0 \\
Earth, $\psi_{\min }^{\odot}=35^{\circ}$ & 3.85 & 0.0 & 0.0 & 0.0 & 0.0 & 0.0 & 0.0 & 0.0 & 0.0 \\
Moon* & 26.2 & 0.0 & 0.0 & 3.06 & 0.001 & 0.001 & 0.001 & 0.0 & 0.0 \\
Moon, $\psi_{\min }^{\odot}=35^{\circ}$ & 0.092 & 0.0 & 0.0 & 0.0 & 0.0 & 0.0 & 0.0 & 0.0 & 0.0 \\
Mars & 116 & 0.0 & 0.0 & 257 & 0.006 & 0.006 & 0.033 & 0.022 & 0.0 \\
Jupiter & 16300 & 0.001 & 0.002 & 21300 & 0.139 & 0.139 & 0.202 & 0.035 & 0.002 \\
Saturn & 5780 & 0.0 & 0.0 & 31300 & 0.020 & 0.020 & 0.035 & 0.008 & 0.0 \\
Uranus & 2080 & 0.0 & 0.0 & 3550 & 0.003 & 0.003 & 0.009 & 0.003 & 0.0 \\
Neptune & 2530 & 0.0 & 0.0 & 3700 & 0.002 & 0.002 & 0.007 & 0.003 & 0.0 \\
\hline
\end{tabular}

as $10^{-3} \mu$ as $\approx 5 \times 10^{-15}$, it is not sufficient to perform the computations using standard "double" 64-bit arithmetic which provides only 16 decimal digits. Routinely, we have used 80bit arithmetic on an Intel processor (18 decimal digits). Some additional accuracy checks have been performed using 128-bit arithmetic on an Ultra-Sparc processor (34 decimal digits) and have shown that the 80-bit arithmetic does not produce substantial roundoff errors and is sufficient for our purposes. Note that since the simulations took several weeks of computing time on a Pentium III processor running at $600 \mathrm{MHz}$ (about one million photon trajectories for each gravitating body were checked), it was hardly possible to perform the simulations within a reasonable time using a software environment emulating arithmetic with arbitrary precision (Maple, Mathematica, etc.).

For the numerical integrations of both the postMinkowskian and post-Newtonian differential equations of motion we have used the Everhart integrator described, e.g. in Everhart $(1974,1985)$. In our program the Everhard integrators of the orders 7, 11, 13, 15, 19, 23 and 27 are implemented, and the internal coefficients of the integrator are coded with an accuracy consistent with the 128-bit arithmetic. This makes it possible to perform the numerical integration with very high precision (at least, up to 34 decimal digits) in a quite efficient way. Our investigation showed that the number 
Table 4. The maximal difference between the models in the simulations with realistic trajectories $\boldsymbol{x}_{\mathrm{A}}^{\mathrm{eph}}(t)$ of the gravitating bodies taken from the JPL planetary ephemeris DE405/LE405. All quantities are given in $\mu$ as. See caption of Table 2 and Sect. 3 for further details.

\begin{tabular}{lrrrrrrrrr}
\hline \hline body & $\delta$ & $\delta^{2}$ & $p M$ & $p N, P_{1}$ & $p N, P_{2}$ & $p N, P_{3}$ & $p N, P_{3}^{\prime}$ & $p N, L_{1}$ & $p N, L_{2}$ \\
\hline Sun & 13000 & 0.001 & 0.001 & 0.002 & 0.001 & 0.001 & 0.001 & 0.001 & 0.001 \\
Mercury & 0.012 & 0.0 & 0.0 & 0.0 & 0.0 & 0.0 & 0.0 & 0.0 & 0.0 \\
Venus & 493 & 0.0 & 0.0 & 30000 & 0.058 & 0.058 & 0.147 & 0.149 & 0.0 \\
Earth & 4.75 & 0.0 & 0.0 & 0.001 & 0.0 & 0.0 & 0.0 & 0.0 & 0.0 \\
Moon & 0.125 & 0.0 & 0.0 & 0.0 & 0.0 & 0.0 & 0.0 & 0.0 & 0.0 \\
Mars & 116 & 0.0 & 0.0 & 385 & 0.008 & 0.008 & 0.040 & 0.025 & 0.0 \\
Jupiter & 16300 & 0.001 & 0.002 & 19600 & 0.175 & 0.175 & 0.255 & 0.038 & 0.002 \\
Saturn & 5780 & 0.0 & 0.0 & 27900 & 0.030 & 0.030 & 0.052 & 0.008 & 0.0 \\
Uranus & 2080 & 0.0 & 0.0 & 3550 & 0.004 & 0.004 & 0.014 & 0.003 & 0.0 \\
Neptune & 2530 & 0.0 & 0.0 & 3700 & 0.002 & 0.002 & 0.008 & 0.003 & 0.0 \\
\hline
\end{tabular}

of internal iterations within the integrator can be chosen to be as low as 1 (or at most 2) without any loss of the resulting accuracy. This can be understood as a consequence of almost straight trajectories of the light. The integrator of order 19 was found to be the most efficient for our calculations. The final global accuracy of the numerical integrations was controlled by integrating the solution backwards. The integration is repeated automatically with higher accuracy parameters of the integrator until the required goal accuracy is reached.

The most time-consuming part of the calculations is the numerical integration of the post-Minkowskian differential equations of light propagation given in Appendix C.2. In order to maximally speed up the numerical integration the code calculating the right-hand side of (C.11) has been optimized using the Maple package CODEGEN (Char et al. 1993). This optimization reduced the number of necessary float-point operations roughly by a factor of 2 .

\section{Results of the simulations}

The maximal differences between the vectors $\boldsymbol{n}$ from the numerical post-Minkowskian solution and the various analytical solutions are summarized in Tables $2-4$. The following conclusions from the numerical simulations can be formulated:

1. The results of the three independent simulations are in good agreement with each other. This serves as an internal consistency check of the simulations.

2. The post-Minkowskian analytical model is virtually indistinguishable from the numerical integration of the postMinkowskian differential equations of light propagation and leads to errors of order $0.002 \mu$ as.

3. As expected the naive model for the light propagation involving the post-Newtonian analytical solution for the body being at rest at its position at the moment of observation (the model $P_{1}$ ) is too inaccurate and leads to errors exceeding 1 mas. Note that our software code for the model $P_{1}$ does not check if the formally calculated impact parameter of the light rays exceeds the radius of the body (such a check is, of course, easy to implement, but if the software detects that the formal impact parameter is smaller than the radius of the body, this can serve only as an evidence of the inaccuracy of the model $P_{1}$ and the situation cannot be corrected within the model $P_{1}$ ). This is the reason why the errors for the model $P_{1}$ given in Tables $2-4$ sometimes exceed the maximal possible gravitational light deflection due to the corresponding body.

4. The post-Newtonian analytical solution for the body being at rest at its position at the moment of closest approach $\left(P_{2}\right)$ or at the retarded moment of time $\left(P_{3}\right)$ are virtually indistinguishable from each other for the solar system applications (e.g., for Jupiter the maximal difference of these models does not exceed $\left.7.5 \times 10^{-4} \mu \mathrm{as}\right)$.

5. Any of these two models $\left(P_{2}\right.$ and $\left.P_{3}\right)$ allows one to attain an accuracy of $\sim 0.18 \mu$ as for the realistic trajectories of the gravitating bodies (Table 4 ). The errors of $\sim 0.75 \mu$ as appearing in the simulation with parabolic trajectories also follow from a simplified analytical considerations and are quoted e.g. in Table 1 of Klioner (2003a) as upper estimates of the effects. The reason for the discrepancy between these estimates for the parabolic trajectories and those for the realistic trajectories were already discussed by Klioner (2003a, p. 1590, above Eq. (34)) where the realistic values for Jupiter and Saturn were predicted. One can see that the predicted realistic values and the values from Table 4 are in good agreement.

6. Simplified calculation of the retarded moment of time as given by (5) (model $P_{3}^{\prime}$ ) increases the errors in the light deflection. The errors attains $\sim 0.3 \mu$ as for the realistic trajectories of the gravitating bodies. Additional simulations show that the following formula gives the retarded moment with sufficient accuracy (this represents one Newtonian iteration for Eq. (4))

$t^{* \prime \prime}=t-\frac{|\rho|^{2}}{c|\rho|-\dot{x}_{\mathrm{A}}^{\mathrm{eph}}(t) \cdot \rho}, \quad \rho=x_{\mathrm{p}}(t)-x_{\mathrm{A}}^{\mathrm{eph}}(t)$.

A solution $P_{3}^{\prime \prime}$ which is similar to $P_{3}^{\prime}$, but with $t_{\text {ref }}=t^{* \prime \prime}$ has the same errors (within the level of $0.001 \mu \mathrm{as}$ ) as the solution $P_{3}$ with exact value of the retarded moment $t_{\text {ref }}=t^{*}$.

7. The post-Newtonian analytical model for a body moving with a constant velocity is indistinguishable from the postMinkowskian model within the accuracy of $0.002 \mu$ as provided that the position and velocity of the body on the rectilinear model trajectory of the body coincide with the actual 
positions and velocities of the body at the moment of the closest approach (model $L_{2}$ ).

8. If the position and velocity of the body on the rectilinear trajectory coincide with the actual positions and velocities at the moment of observation (model $L_{1}$ ) the error exceeds $0.1 \mu$ as.

Let us also note that increasing the minimal Sun avoidance angle $\psi_{\min }^{\odot}$ would reduce the errors for the Sun, Mercury, Venus, Earth and the Moon (all the gravitating bodies which are closer to the Sun than the observer). Therefore, the numbers in Tables 2-4 can be considered as upper estimates for $\psi_{\min }^{\odot} \geq 35^{\circ}$.

\section{Concluding remarks}

The results of our numerical simulations are in good agreement with the theoretical discussion by Klioner (2003a). These results allows one to formulate the following practical recommendations for data processing of microarcsecond positional observations performed from the solar system:

- If an accuracy of $0.2 \mu$ as is sufficient one can employ the simple post-Newtonian analytical model for the light propagation in the gravitational field of a motionless body and substitute in that model the actual position of the body evaluated either at the moment of closest approach $t^{\mathrm{ca}}$ or at the retarded moment $t^{*}$. If $t^{*}$ is used, Eq. (6) can be employed to calculate it with sufficient accuracy.

- If an accuracy below $0.2 \mu$ as is required one should use either the full post-Minkowskian analytical model as given in Appendix C.4 (neglecting the integral $\boldsymbol{g}\left(t_{0}, t\right)$ in (C.30)) or the post-Newtonian analytical model for a body moving with a constant velocity (Appendix B.4) and choose the constants $\boldsymbol{x}_{\mathrm{A} 0}$ and $\boldsymbol{v}_{\mathrm{A}}$ of that model to coincide with the actual position and velocity of the body at $t^{\mathrm{ca}}$.

\section{Appendix A: Equations of null geodesics}

Here we summarize the formulas for the null geodesics in a weak gravitational field used in this paper. The metric tensor $g_{\alpha \beta}$ is supposed to have the form

$g_{\alpha \beta}=\eta_{\alpha \beta}+h_{\alpha \beta}$,

where

$\eta_{\alpha \beta}=\operatorname{diag}(-1,+1,+1,+1)$

is the flat Minkowski metric and $h_{\alpha \beta}=h_{\alpha \beta}(t, \boldsymbol{x})$ is the nonGalilean part of the metric.

\section{A.1. Post-Newtonian approximation}

First, we use the standard post-Newtonian assumptions on the orders of magnitude of $h_{\alpha \beta}$ with respect to the formal parameter $c^{-1}$ :

$h_{00}=O\left(c^{-2}\right)$,

$h_{0 i}=O\left(c^{-3}\right)$,

$h_{i j}=O\left(c^{-2}\right)$.
Then the equations of null geodesics in the first post-Newtonian approximation read

$$
\begin{aligned}
\ddot{x}^{i}= & \frac{1}{2} c^{2} h_{00, i}-h_{00, k} \dot{x}^{k} \dot{x}^{i}-\left(h_{i k, l}-\frac{1}{2} h_{k l, i}\right) \dot{x}^{k} \dot{x}^{l}-\frac{1}{2} h_{00, t} \dot{x}^{i} \\
& -\left(\frac{1}{c} h_{0 k, j}-\frac{1}{2 c^{2}} h_{j k, t}\right) \dot{x}^{j} \dot{x}^{k} \dot{x}^{i}-c\left(h_{0 i, k}-h_{0 k, i}\right) \dot{x}^{k} \\
& -h_{i k, t} \dot{x}^{k}+O\left(c^{-2}\right)
\end{aligned}
$$

where for any small latin index $i$ except for $t$ one has $A_{, i}=\frac{\partial}{\partial x^{i}} A$ and $A_{, t}=\frac{\partial}{\partial t} A$. Note that the velocity of the photon $\dot{x}^{k}=O(c)$. Equation (A.4) is valid in the post-Newtonian approximation which is also called weak-field-slow-motion approximation. The assumption $h_{0 i}=O\left(c^{-3}\right)$ means that the velocity of the gravitating bodies are considered to be small with respect to $c$. Therefore, these equations cannot be used if the velocities of the gravitating bodies are large.

\section{A.2. Post-Minkowskian approximation}

Second, we use the post-Minkowskian properties of the metric tensor and consider all the components of $h_{\alpha \beta}$ to be of first order with respect to gravitational constant $G$ :

$h_{00}=O(G)$,

$h_{0 i}=O(G)$,

$h_{i j}=O(G)$.

No expansion in terms of $c^{-1}$ is used here. Then, one has

$$
\begin{aligned}
\ddot{x}^{i}= & \frac{1}{2} c^{2} h_{00, i}-h_{00, k} \dot{x}^{k} \dot{x}^{i}-\left(h_{i k, l}-\frac{1}{2} h_{k l, i}\right) \dot{x}^{k} \dot{x}^{l}-\frac{1}{2} h_{00, t} \dot{x}^{i} \\
& -\left(\frac{1}{c} h_{0 k, j}-\frac{1}{2 c^{2}} h_{j k, t}\right) \dot{x}^{j} \dot{x}^{k} \dot{x}^{i}-c\left(h_{0 i, k}-h_{0 k, i}\right) \dot{x}^{k} \\
& -h_{i k, t} \dot{x}^{k}-c h_{0 i, t}+O\left(G^{2}\right) .
\end{aligned}
$$

The only formal difference between (A.6) and (A.4) is the last term $-c h_{0 i, t}$ which has first order with respect to $G$, but is $O\left(c^{-2}\right)$ and has been omitted in (A.4). Equation (A.6) has been derived without any assumption on the velocity of the bodies. This equation is valid in the first post-Minkowskian approximation which is also called weak-field limit.

\section{A.3. Initial-value problem for the equations of motion}

Initial value problem for the differential Eqs. (A.4) and (A.6) can be formulated as

$\boldsymbol{x}\left(t_{0}\right)=\boldsymbol{x}_{0}$,

$\dot{\boldsymbol{x}}\left(t_{0}\right)=c \boldsymbol{\mu} s\left(t_{0}\right), \quad \boldsymbol{\mu} \cdot \boldsymbol{\mu}=1$.

Function $s$ can be generally determined from the condition of the geodesic to be a null one:

$g_{\alpha \beta} k^{\alpha} k^{\beta}=0, \quad k^{\alpha}=\left(1, \frac{1}{c} \dot{x}\right)$, 
which for metric (A.1) gives

$$
s=\frac{\sqrt{\left(1-h_{00}\right)\left(1+h_{i j} \mu^{i} \mu^{j}\right)}-h_{0 i} \mu^{i}}{1+h_{i j} \mu^{i} \mu^{j}} .
$$

Note that $s$ is a function of time and position (via the metric components $h_{\alpha \beta}$ ) as well as of the direction $\mu$, but for a given trajectory of the photon it can be considered as a function of time.

Taking into account the orders of magnitude of $h_{\alpha \beta}$ given by (A.3) in the first post-Newtonian approximation one gets

$$
s=1-\frac{1}{2} h_{00}-\frac{1}{2} h_{i j} \mu^{i} \mu^{j}-h_{0 i} \mu^{i}+O\left(c^{-4}\right) .
$$

It is easy to see that this expression is valid also in the first post-Minkowskian approximation (that is, it contains all terms of (A.9) linear with respect to $G$ ).

\section{Appendix B: Light propagation in the post-Newtonian approximation}

\section{B.1. Metric tensor}

The non-Galilean components of the metric tensor in the postNewtonian approximation read

$$
\begin{aligned}
& h_{00}=\frac{2}{c^{2}} w(t, \boldsymbol{x})+O\left(c^{-4}\right), \\
& h_{0 i}=-\frac{4}{c^{3}} w^{i}(t, \boldsymbol{x})+O\left(c^{-5}\right), \\
& h_{i j}=\frac{2}{c^{2}} \delta_{i j} w(t, \boldsymbol{x})+O\left(c^{-4}\right) .
\end{aligned}
$$

For moving mass monopoles the potentials have the form

$$
\begin{aligned}
w & =\sum_{\mathrm{A}} \frac{G M_{\mathrm{A}}}{r_{\mathrm{A}}}, \\
w^{i} & =\sum_{\mathrm{A}} \frac{G M_{\mathrm{A}}}{r_{\mathrm{A}}} \dot{x}_{\mathrm{A}}^{i}(t),
\end{aligned}
$$

where $r_{\mathrm{A}}=\left|\boldsymbol{r}_{\mathrm{A}}\right|$,

$$
\boldsymbol{r}_{\mathrm{A}}(t, \boldsymbol{x})=\boldsymbol{x}-\boldsymbol{x}_{\mathrm{A}}(t),
$$

$\boldsymbol{x}_{\mathrm{A}}(t)$ is the position of body $\mathrm{A}$, and $\dot{\boldsymbol{x}}_{\mathrm{A}}(t)$ is the velocity of body A.

The metric (B.1) with potentials (B.2)-(B.3) coincides with the metric tensor of the Barycentric Celestial Reference System given by the IAU (2001) (see also Soffel et al. 2003 for a discussion). The higher-order multipole moments of the bodies' gravitational fields (B.2)-(B.3) also discussed in IAU (2001) will be ignored in this paper.

\section{B.2. Differential equations of light propagation}

The post-Newtonian equations of motion of a photon then read

$\ddot{\boldsymbol{x}}=\sum_{\mathrm{A}} \frac{G M_{\mathrm{A}}}{r_{\mathrm{A}}^{2}}\left(A_{\mathrm{A}} \boldsymbol{n}_{\mathrm{A}}+B_{\mathrm{A}} \boldsymbol{v}+C_{\mathrm{A}} \boldsymbol{v}_{\mathrm{A}}\right)+O\left(c^{-2}\right)$,

$A_{\mathrm{A}}=2+\gamma-4 \delta$

$$
\begin{aligned}
& B_{\mathrm{A}}=4(1-\alpha) \delta-(1-\beta)(2+\gamma), \\
& C_{\mathrm{A}}=-4(1-\alpha), \\
& \alpha=1-\boldsymbol{n}_{\mathrm{A}} \cdot \boldsymbol{v}, \\
& \beta=1-\boldsymbol{n}_{\mathrm{A}} \cdot \boldsymbol{v}_{\mathrm{A}}, \\
& \gamma=1-\boldsymbol{v} \cdot \boldsymbol{v}, \\
& \delta=1-\boldsymbol{v} \cdot \boldsymbol{v}_{\mathrm{A}},
\end{aligned}
$$

where $\boldsymbol{n}_{\mathrm{A}}=\boldsymbol{r}_{\mathrm{A}} / r_{\mathrm{A}}, \boldsymbol{v}=\dot{\boldsymbol{x}} / c, \boldsymbol{v}_{\mathrm{A}}=\dot{\boldsymbol{x}}_{\mathrm{A}} / c$.

\section{B.3. Initial-value problem}

From the general formulas of Sect. A.3 the initial-value problem for (B.5)-(B.12) reads

$\boldsymbol{x}\left(t_{0}\right)=\boldsymbol{x}_{0}$,

$\dot{\boldsymbol{x}}\left(t_{0}\right)=c \boldsymbol{\mu} s\left(t_{0}\right), \quad \boldsymbol{\mu} \cdot \boldsymbol{\mu}=1$,

and

$s(t)=1-\frac{2}{c^{2}} \sum_{\mathrm{A}} \frac{G M_{\mathrm{A}}}{r_{\mathrm{A}}(t)}\left(1-2 \boldsymbol{\mu} \cdot \boldsymbol{v}_{\mathrm{A}}(t)\right)+O\left(c^{-4}\right)$.

\section{B.4. Analytical solution for a body in uniform motion}

Two analytical solutions of Eqs. (B.5)-(B.12) and (B.13)-(B.14) are known: 1) the classical solution for a body at rest, i.e. $\boldsymbol{x}_{\mathrm{A}}=$ const; and 2) a solution for a body moving with a constant velocity. The first solution is clearly contained in the second one. An approximate analytical solution for bodies having a constant velocity, that is for $\boldsymbol{x}_{\mathrm{A}}(t)=\boldsymbol{x}_{\mathrm{A} 0}+\boldsymbol{v}_{\mathrm{A}}\left(t-t_{0}\right)$, where $\boldsymbol{x}_{\mathrm{A} 0}=$ const and $\boldsymbol{v}_{\mathrm{A}}=$ const, has been first derived by Klioner (1989) (see also Klioner \& Kopeikin 1992). The solution reads

$$
\begin{aligned}
\boldsymbol{x}(t)= & \boldsymbol{x}\left(t_{0}\right)+c \boldsymbol{\mu} s\left(t_{0}\right)\left(t-t_{0}\right)+\Delta \boldsymbol{x}\left(t_{0}, t\right) \\
& -\Delta \dot{\boldsymbol{x}}\left(t_{0}\right)\left(t-t_{0}\right),
\end{aligned}
$$

$\frac{1}{c} \dot{\boldsymbol{x}}(t)=\boldsymbol{\mu} s\left(t_{0}\right)+\frac{1}{c} \Delta \dot{\boldsymbol{x}}(t)-\frac{1}{c} \Delta \dot{\boldsymbol{x}}\left(t_{0}\right)$,

$\Delta \boldsymbol{x}\left(t_{0}, t\right)=-\sum_{\mathrm{A}} \frac{2 G M_{\mathrm{A}}}{c^{2}}\left(\boldsymbol{d}_{\mathrm{A}} \mathcal{I}_{\mathrm{A}}+\boldsymbol{g}_{\mathrm{A}} \mathcal{J}_{\mathrm{A}}\right)+O\left(c^{-4}\right)$,

$\boldsymbol{d}_{\mathrm{A}}=\boldsymbol{\mu} \times\left(\boldsymbol{r}_{\mathrm{A} 0} \times \boldsymbol{g}_{\mathrm{A}}\right)$,

$\boldsymbol{g}_{\mathrm{A}}=\boldsymbol{\mu}-\boldsymbol{v}_{\mathrm{A}}$,

$\mathcal{I}_{\mathrm{A}}=\frac{1}{\left|\boldsymbol{g}_{\mathrm{A}}\right|\left|\boldsymbol{r}_{\mathrm{A}}\right|-\boldsymbol{g}_{\mathrm{A}} \cdot \boldsymbol{r}_{\mathrm{A}}}-\frac{1}{\left|\boldsymbol{g}_{\mathrm{A}}\right|\left|\boldsymbol{r}_{\mathrm{A} 0}\right|-\boldsymbol{g}_{\mathrm{A}} \cdot \boldsymbol{r}_{\mathrm{A} 0}}$,

$\mathcal{J}_{\mathrm{A}}=\log \frac{\left|\boldsymbol{g}_{\mathrm{A}}\right|\left|\boldsymbol{r}_{\mathrm{A}}\right|+\boldsymbol{g}_{\mathrm{A}} \cdot \boldsymbol{r}_{\mathrm{A}}}{\left|\boldsymbol{g}_{\mathrm{A}}\right|\left|\boldsymbol{r}_{\mathrm{A} 0}\right|+\boldsymbol{g}_{\mathrm{A}} \cdot \boldsymbol{r}_{\mathrm{A} 0}}$

$\boldsymbol{r}_{\mathrm{A}}(t)=\boldsymbol{x}(t)-\boldsymbol{x}_{\mathrm{A}}(t)$,

$\boldsymbol{r}_{\mathrm{A} 0}=\boldsymbol{x}\left(t_{0}\right)-\boldsymbol{x}_{\mathrm{A}}\left(t_{0}\right)$, 
$\frac{1}{c} \Delta \dot{\boldsymbol{x}}(t)=-\sum_{\mathrm{A}} \frac{2 G M_{\mathrm{A}}}{c^{2}}\left(\boldsymbol{d}_{\mathrm{A}} \frac{1}{c} \dot{\mathcal{I}}_{\mathrm{A}}+\boldsymbol{g}_{\mathrm{A}} \frac{1}{c} \dot{\mathcal{J}}_{\mathrm{A}}\right)+O\left(c^{-4}\right)$,

$\frac{1}{c} \dot{\mathcal{I}}_{\mathrm{A}}=\frac{\left|\boldsymbol{g}_{\mathrm{A}}\right|}{\left|\boldsymbol{r}_{\mathrm{A}}\right|\left(\left|\boldsymbol{g}_{\mathrm{A}}\right|\left|\boldsymbol{r}_{\mathrm{A}}\right|-\boldsymbol{g}_{\mathrm{A}} \cdot \boldsymbol{r}_{\mathrm{A}}\right)}$,

$\frac{1}{c} \dot{\mathcal{J}}_{\mathrm{A}}=\frac{\left|\boldsymbol{g}_{\mathrm{A}}\right|}{\left|\boldsymbol{r}_{\mathrm{A}}\right|}$

This solution has two parameters $\boldsymbol{x}_{\mathrm{A} 0}$ and $\boldsymbol{v}_{\mathrm{A}}$. Note that the values of $\boldsymbol{x}_{\mathrm{A} 0}$ and $\boldsymbol{v}_{\mathrm{A}}$ are arbitrary and it is a priori unclear how to relate $x_{\mathrm{A} 0}$ to the actual trajectory $\boldsymbol{x}_{\mathrm{A}}^{\mathrm{eph}}(t)$ of the body in order to minimize the errors (see Sect. 3).

The classical solution of (B.5)-(B.12) for a body at rest can be easily restored from (B.15)-(B.26) by setting $\boldsymbol{v}_{\mathrm{A}}=0$ that implies $\boldsymbol{g}_{\mathrm{A}}=\boldsymbol{\mu}$ and $\left|\boldsymbol{g}_{\mathrm{A}}\right|=1$.

\section{Appendix C: Theoretical results in the post-Minkowskian approximation}

\section{C.1. Metric tensor}

In the post-Minkowskian approximation the metric tensor of a system of arbitrarily moving mass monopoles can be written (see Kopeikin \& Schäfer 1999; Kopeikin \& Mashhoon 2002) with retarded potentials similar to the Lienard-Wiechert potentials which are well known from the classical electrodynamics (Jackson 1974). The metric reads

$h_{00}=\frac{2}{c^{2}} \mathcal{W}(t, x)+O\left(G^{2}\right)$,

$h_{0 i}=-\frac{4}{c^{3}} \boldsymbol{W}^{i}(t, \boldsymbol{x})+O\left(G^{2}\right)$,

$h_{i j}=\frac{2}{c^{2}} \mathcal{W}^{i j}(t, \boldsymbol{x})+O\left(G^{2}\right)$,

$\mathcal{W}(t, \boldsymbol{x})=\sum_{\mathrm{A}} \frac{G M_{\mathrm{A}}}{r_{\mathrm{A}}^{*} \beta_{*}}\left(2 \Gamma_{*}-\Gamma_{*}^{-1}\right)$,

$\mathcal{W}^{i}(t, \boldsymbol{x})=\sum_{\mathrm{A}} \frac{G M_{\mathrm{A}}}{r_{\mathrm{A}}^{*} \beta_{*}} \Gamma_{*} c v_{\mathrm{A}}^{* i}$,

$\mathcal{W}^{i j}(t, \boldsymbol{x})=\sum_{\mathrm{A}} \frac{G M_{\mathrm{A}}}{r_{\mathrm{A}}^{*} \beta_{*}}\left(\delta^{i j} \Gamma_{*}^{-1}+2 \Gamma_{*} v_{\mathrm{A}}^{* i} v_{\mathrm{A}}^{* j}\right)$,

where $\boldsymbol{r}_{\mathrm{A}}^{*}=\boldsymbol{x}-\boldsymbol{x}_{\mathrm{A}}^{*}, r_{\mathrm{A}}^{*}=\left|\boldsymbol{r}_{\mathrm{A}}^{*}\right|, \boldsymbol{x}_{\mathrm{A}}^{*}=\boldsymbol{x}_{\mathrm{A}}\left(t_{\mathrm{A}}^{*}\right), \boldsymbol{v}_{\mathrm{A}}^{*}=\frac{\mathrm{d}}{\mathrm{d} t_{\mathrm{A}}^{*}} \boldsymbol{x}_{\mathrm{A}}^{*} / c$, $\boldsymbol{n}_{\mathrm{A}}^{*}=\boldsymbol{r}_{\mathrm{A}}^{*} / r_{\mathrm{A}}^{*}, \beta_{*}=1-\boldsymbol{n}_{\mathrm{A}}^{*} \cdot \boldsymbol{v}_{\mathrm{A}}^{*}, \Gamma_{*}=\left(1-\boldsymbol{v}_{\mathrm{A}}^{*} \cdot \boldsymbol{v}_{\mathrm{A}}^{*}\right)^{-1 / 2}$. Here and below in Appendix. $\mathrm{C}$ the position $\boldsymbol{x}_{\mathrm{A}}$, velocity $\dot{\boldsymbol{x}}_{\mathrm{A}}$ and acceleration $\ddot{\boldsymbol{x}}_{\mathrm{A}}$ of the gravitating bodies are computed at the moment $t_{\mathrm{A}}^{*}=t_{\mathrm{A}}^{*}(t, \boldsymbol{x})$ being the retarded moment of time defined by the following implicit equation

$t_{\mathrm{A}}^{*}+\frac{1}{c} r_{\mathrm{A}}^{*}=t$

Note that $r_{\mathrm{A}}(t)=r_{\mathrm{A}}^{*} \beta_{*}+O\left(r_{\mathrm{A}}^{2} / c^{2}\right)$, so that formally $W=$ $w+O\left(c^{-2}\right), \mathcal{W}^{i}=w^{i}+\mathcal{O}\left(c^{-2}\right), \mathcal{W}^{i j}=\delta^{i j} w+O\left(c^{-2}\right)$, and Eqs. (B.1)-(B.4) can be easily restored from (C.1)-(C.4) within the first post-Newtonian approximation.

\section{C.2. Differential equations of light propagation}

Substituting (C.1) into (A.6) one gets

$$
\begin{aligned}
& \ddot{\boldsymbol{x}}=\boldsymbol{W}_{, i}-\frac{2}{c^{2}} \boldsymbol{W}_{, k} \dot{x}^{k} \dot{x}^{i}-\frac{2}{c^{2}} \mathcal{W}_{, l}^{i k} \dot{x}^{k} \dot{x}^{l}+\frac{1}{c^{2}} \mathcal{W}_{, i}^{k l} \dot{x}^{k} \dot{x}^{l} \\
& -\frac{1}{c^{2}} \boldsymbol{W}_{, t} \dot{x}^{i}+\frac{4}{c^{4}} \boldsymbol{W}_{, j}^{k} \dot{x}^{j} \dot{x}^{k} \dot{x}^{i}+\frac{1}{c^{4}} \boldsymbol{W}^{j k}{ }_{, t} \dot{x}^{j} \dot{x}^{k} \dot{x}^{i} \\
& +\frac{4}{c^{2}} \boldsymbol{W}_{, k}^{i} \dot{x}^{k}-\frac{4}{c^{2}} \boldsymbol{W}_{, i}^{k} \dot{x}^{k}-\frac{2}{c^{2}} \boldsymbol{W}^{i k}{ }_{, t} \dot{x}^{k} \\
& +\frac{4}{c^{2}} W_{, t}^{i}+O\left(G^{2}\right) \text {. }
\end{aligned}
$$

Here, as usual, $A_{, t}=\frac{\partial}{\partial t} A$ is the partial derivative with respect to $t$, and for any latin index $i$ with except for $t$ one has $A_{, i}=\frac{\partial}{\partial x^{i}} A$. Since the potentials $\mathcal{W}, \mathcal{W}^{i}$ and $\mathcal{W}^{i j}$ are given by Eqs. (C.2)-(C.4) explicitly as functions of the retarded time $t_{\mathrm{A}}^{*}=t_{\mathrm{A}}^{*}(t, \boldsymbol{x})$ one has to use

$$
\begin{aligned}
& \frac{\partial}{\partial t} A(t, \boldsymbol{x})=\frac{\partial A\left(t_{\mathrm{A}}^{*}, \boldsymbol{x}\right)}{\partial t_{\mathrm{A}}^{*}} \frac{\partial t_{\mathrm{A}}^{*}}{\partial t}, \\
& \frac{\partial}{\partial x^{i}} A(t, \boldsymbol{x})=\frac{\partial A\left(t_{\mathrm{A}}^{*}, \boldsymbol{x}\right)}{\partial t_{\mathrm{A}}^{*}} \frac{\partial t_{\mathrm{A}}^{*}}{\partial x^{i}}+\frac{\partial A\left(t_{\mathrm{A}}^{*}, \boldsymbol{x}\right)}{\partial x^{i}},
\end{aligned}
$$

where from (C.5)

$$
\begin{aligned}
& \frac{\partial t_{\mathrm{A}}^{*}}{\partial t}=\beta_{*}^{-1}, \\
& \frac{\partial t_{\mathrm{A}}^{*}}{\partial x^{i}}=-c^{-1} \beta_{*}^{-1} n_{\mathrm{A}}^{* i} .
\end{aligned}
$$

Substituting (C.2)-(C.4) into (C.6) and using (C.7)-(C.8) one gets the explicit form of the equations of motion of a photon in the first post-Minkowskian approximation

$$
\begin{aligned}
\ddot{\boldsymbol{x}}= & \sum_{\mathrm{A}} \frac{G M_{\mathrm{A}} \Gamma_{*}^{3}}{r_{\mathrm{A}}^{* 2} \beta_{*}^{3}}\left(\mathcal{A}_{\mathrm{A}} \boldsymbol{n}_{\mathrm{A}}^{*}+\mathcal{B}_{\mathrm{A}} \boldsymbol{v}+C_{\mathrm{A}} \boldsymbol{v}_{\mathrm{A}}^{*}+\mathcal{D}_{\mathrm{A}} \boldsymbol{a}_{\mathrm{A}}^{*}\right) \\
& +O\left(G^{2}\right)
\end{aligned}
$$

$\mathcal{A}_{\mathrm{A}}=\left[\Gamma_{*}^{-2} \gamma-2 \delta_{*}^{2}\right] \Gamma_{*}^{-2}\left(\Gamma_{*}^{-2}+\varepsilon_{*}\right)$ $-\left[\Gamma_{*}^{-2} \gamma+2 \delta_{*}^{2}\right] \eta_{*} \beta_{*}+4 \zeta_{*} \Gamma_{*}^{-2} \beta_{*} \delta_{*}$,

$\mathcal{B}_{\mathrm{A}}=\Gamma_{*}^{-2}\left[-\Gamma_{*}^{-4} \gamma-\Gamma_{*}^{-2}\left\{2 \delta_{*}\left(2 \alpha_{*}-\delta_{*}\right)+\left(\epsilon_{*}-\beta_{*}\right) \gamma\right\}\right.$

$$
\left.+2 \delta_{*}\left\{\beta_{*} \delta_{*}-\epsilon_{*}\left(2 \alpha_{*}-\delta_{*}\right)\right\}+4 \zeta_{*} \beta_{*}\left(\alpha_{*}-\delta_{*}\right)\right]
$$$$
+\eta_{*} \beta_{*}\left(\Gamma_{*}^{-2} \gamma-2 \delta_{*}\left(2 \alpha_{*}-\delta_{*}\right)\right),
$$

$C_{\mathrm{A}}=\Gamma_{*}^{-4}\left[4 \delta_{*} \alpha_{*}-\beta_{*} \gamma\right]$

$$
\begin{aligned}
& +2 \Gamma_{*}^{-2}\left[\delta_{*}\left\{2 \epsilon_{*} \alpha_{*}-\beta_{*} \delta_{*}\right\}-2 \zeta_{*} \beta_{*} \alpha_{*}\right] \\
& +4 \eta_{*} \alpha_{*} \beta_{*} \delta_{*},
\end{aligned}
$$

$\mathcal{D}_{\mathrm{A}}=4 \Gamma_{*}^{-2} \alpha_{*} \beta_{*} \delta_{*} c^{-1} r_{\mathrm{A}}^{*}$,

$\alpha_{*}=1-\boldsymbol{n}_{\mathrm{A}}^{*} \cdot \boldsymbol{v}$

$\beta_{*}=1-\boldsymbol{n}_{\mathrm{A}}^{*} \cdot \boldsymbol{v}_{\mathrm{A}}^{*}$,

$\gamma=1-\boldsymbol{v} \cdot \boldsymbol{v}$, 
$\delta_{*}=1-\boldsymbol{v} \cdot \boldsymbol{v}_{\mathrm{A}}^{*}$,

$\boldsymbol{\varepsilon}_{*}=\left(\boldsymbol{a}_{\mathrm{A}}^{*} \cdot \boldsymbol{n}_{\mathrm{A}}^{*}\right) c^{-1} r_{\mathrm{A}}^{*}$,

$\zeta_{*}=\left(\boldsymbol{a}_{\mathrm{A}}^{*} \cdot \boldsymbol{v}\right) c^{-1} r_{\mathrm{A}}^{*}$,

$\eta_{*}=\left(\boldsymbol{a}_{\mathrm{A}}^{*} \cdot \boldsymbol{v}_{\mathrm{A}}^{*}\right) c^{-1} r_{\mathrm{A}}^{*}$,

and $\boldsymbol{v}=c^{-1} \frac{\mathrm{d}}{\mathrm{d} t} \boldsymbol{x}, \boldsymbol{a}_{\mathrm{A}}^{*}=\frac{\mathrm{d}}{\mathrm{d} t_{\mathrm{A}}^{*}} \boldsymbol{v}_{\mathrm{A}}^{*}=c^{-1} \frac{\mathrm{d}^{2}}{\mathrm{~d} t_{\mathrm{A}}^{* 2}} \boldsymbol{x}_{\mathrm{A}}\left(t_{\mathrm{A}}^{*}\right)$.

Note that if the accelerations $\boldsymbol{a}_{\mathrm{A}}^{*}$ are considered to be of gravitational nature (and, therefore, $a_{\mathrm{A}}^{*}=O(G)$ ) the terms in (C.11) containing $\boldsymbol{a}_{\mathrm{A}}^{*}$ are of order $O\left(G^{2}\right)$ and can be discarded in the first post-Minkowskian approximation. However, in our theory no equations of motion of the gravitating bodies are considered, so that the accelerations $\boldsymbol{a}_{\mathrm{A}}^{*}$ can be of any nature and are considered to be given. That is why we retain the accelerations $\boldsymbol{a}_{\mathrm{A}}^{*}$ in (C.11) in the first post-Minkowskian approximation.

From Eqs. (C.11)-(C.22) one can restore the postNewtonian equations of motion (B.5)-(B.12) neglecting in (C.11)-(C.22) all the terms formally of order $O\left(c^{-2}\right)$ (e.g., all the acceleration-dependent terms) and taking into account that in (C.11)-(C.22) the position of the bodies are calculated at the retarded moment of time $t_{\mathrm{A}}^{*}$ while in (B.5)-(B.12) at moment $t$. The latter circumstance gives the following relations: $\boldsymbol{r}_{\mathrm{A}}(t)=\boldsymbol{r}_{\mathrm{A}}^{*}-r_{\mathrm{A}}^{*} \boldsymbol{v}_{\mathrm{A}}^{*}+O\left(c^{-2}\right), r_{\mathrm{A}}(t)=r_{\mathrm{A}}^{*} \beta_{*}+O\left(c^{-2}\right)$, $\boldsymbol{n}(t)=\left(\boldsymbol{n}_{\mathrm{A}}^{*}-\boldsymbol{v}_{\mathrm{A}}^{*}\right) / \beta_{*}+O\left(c^{-2}\right)$.

Since from (C.11) it follows that

$\boldsymbol{x}=\boldsymbol{x}_{0}+c \boldsymbol{\mu}\left(t-t_{0}\right)+O(G), \quad \boldsymbol{\mu} \cdot \boldsymbol{\mu}=1$

in the first post-Minkowski an approximation in the right-hand side of Eq. (C.11) one can put

$\boldsymbol{x}=\boldsymbol{x}_{0}+c \boldsymbol{\mu}\left(t-t_{0}\right), \quad \boldsymbol{\mu} \cdot \boldsymbol{\mu}=1$

and, therefore, $\boldsymbol{v}=\boldsymbol{\mu}$, which implies, e.g., $\gamma=0$. Formally, this makes Eq. (C.11) integrable in quadratures (since the righthand side is simply a function of time). We prefer here not to do so and retain Eq. (C.11) in the form of a differential equation as given by (C.6).

\section{C.3. Initial-value problem}

Initial value problem for Eq. (C.11)

$$
\begin{aligned}
& \boldsymbol{x}\left(t_{0}\right)=\boldsymbol{x}_{0}, \\
& \dot{\boldsymbol{x}}\left(t_{0}\right)=c \boldsymbol{\mu} \widetilde{s}\left(t_{0}\right), \quad \boldsymbol{\mu} \cdot \boldsymbol{\mu}=1
\end{aligned}
$$

with

$\widetilde{s}(t)=1-\frac{2}{c^{2}} \sum_{\mathrm{A}} \frac{G M_{\mathrm{A}} \Gamma_{*}}{r_{\mathrm{A}}^{*} \beta_{*}} \theta_{*}^{2}+O\left(G^{2}\right)$,

$\theta_{*}=1-\boldsymbol{\mu} \cdot \boldsymbol{v}_{\mathrm{A}}^{*}$

\section{C.4. Analytical solution in the post-Minkowskian approximation}

The explicit form of the analytical solution in the first postMinkowskian approximation derived by Kopeikin \& Schäfer (1999) reads

$$
\begin{aligned}
\boldsymbol{x}(t)= & \boldsymbol{x}\left(t_{0}\right)+c \boldsymbol{\mu} \widetilde{s}\left(t_{0}\right)\left(t-t_{0}\right)+\widetilde{\Delta} \boldsymbol{x}\left(t_{0}, t\right) \\
& -\Delta \dot{\boldsymbol{x}}\left(t_{0}\right)\left(t-t_{0}\right), \\
\frac{1}{c} \dot{\boldsymbol{x}}(t)= & \boldsymbol{\mu} \widetilde{s}\left(t_{0}\right)+\frac{1}{c} \widetilde{\Delta} \dot{\boldsymbol{x}}(t)-\frac{1}{c} \widetilde{\Delta} \dot{\boldsymbol{x}}\left(t_{0}\right),
\end{aligned}
$$

$$
\begin{aligned}
\widetilde{\Delta} \boldsymbol{x}\left(t_{0}, t\right)= & -\sum_{\mathrm{A}} \frac{2 G M_{\mathrm{A}}}{c^{2}}\left(\boldsymbol{f}(t)-\boldsymbol{f}\left(t_{0}\right)+\boldsymbol{g}\left(t_{0}, t\right)\right) \\
& +O\left(G^{2}\right),
\end{aligned}
$$

$\boldsymbol{f}(t)=\Gamma_{*}\left(\theta_{*} \frac{\boldsymbol{\mu} \times\left(\boldsymbol{r}_{\mathrm{A}}^{*} \times \boldsymbol{\mu}\right)}{r_{\mathrm{A}}^{*} \alpha_{*}}-\left(\boldsymbol{\mu}-\boldsymbol{v}_{\mathrm{A}}^{*}\right) \log \left(r_{\mathrm{A}}^{*} \alpha_{*}\right)\right)$,

$$
\begin{aligned}
\boldsymbol{g}\left(t_{0}, t\right)= & \int_{t_{0}}^{t} \frac{\Gamma_{*}^{3}}{r_{\mathrm{A}}^{*} \beta_{*}}\left[\left(\Gamma_{*}^{2} \zeta_{*}-\theta_{*} \eta_{*}\right) \boldsymbol{\mu} \times\left(\boldsymbol{n}_{\mathrm{A}}^{*} \times \boldsymbol{\mu}\right)+\alpha_{*}\right. \\
& \left.\times \log \left(r_{\mathrm{A}}^{*} \alpha_{*}\right)\left(\eta_{*}\left(\boldsymbol{\mu}-\boldsymbol{v}_{\mathrm{A}}^{*}\right)-\Gamma_{*}^{-2} c^{-1} r_{\mathrm{A}}^{*} \boldsymbol{a}_{\mathrm{A}}^{*}\right)\right] c \mathrm{~d} t
\end{aligned}
$$

$$
\begin{aligned}
\frac{1}{c} \widetilde{\Delta} \dot{\boldsymbol{x}}(t)= & -\sum_{\mathrm{A}} \frac{2 G M_{\mathrm{A}}}{c^{2}} \frac{\Gamma_{*} \theta_{*}}{r_{\mathrm{A}}^{*} \beta_{*}}\left(\theta_{*} \frac{\boldsymbol{\mu} \times\left(\boldsymbol{n}_{\mathrm{A}}^{*} \times \boldsymbol{\mu}\right)}{\alpha_{*}}\right. \\
& \left.+\left(2-\theta_{*}\right) \boldsymbol{\mu}-2 \boldsymbol{v}_{\mathrm{A}}^{*}\right)+O\left(G^{2}\right) .
\end{aligned}
$$

Here $\boldsymbol{g}\left(t_{0}, t\right)$ are integrals depending on the accelerations of the bodies. The integrals can be transformed into integrals with respect to retarded time $t_{\mathrm{A}}^{*}$ as described in Kopeikin \& Schäfer (1999). In the right-hand sides of (C.30) and (C.33) one should put $\boldsymbol{x}(t)=\boldsymbol{x}\left(t_{0}\right)+c \boldsymbol{\mu}\left(t-t_{0}\right)$.

Note that the solution for $\frac{1}{c} \widetilde{\Delta} \dot{\boldsymbol{x}}(t)$ is exact within the first post-Minkowskian approximation (i.e. all the omitted terms are of order $G^{2}$ ). It is remarkable that this solution can be written in such a compact closed form for arbitrary motion of the gravitating bodies. Note also that the solution depends only on the position and velocity of the bodies in a single moment of time - the retarded moment $t_{\mathrm{A}}^{*}$ corresponding to time $t$ - and does not depend on the previous motion of the bodies. If all the terms which are formally of order $O\left(c^{-4}\right)$ (these are the terms at least quadratic with respect to $\boldsymbol{v}_{\mathrm{A}}$ ) are dropped both in (C.33) and (B.24) the two formulas are equivalent. One can also show that Eqs. (C.30)-(C.31) agree with Eqs. (B.17)-(B.21) in the first post-Newtonian approximation provided that $\boldsymbol{v}_{\mathrm{A}}$ is considered to be constant.

Taking into account that for any function $A^{i}$ and $\boldsymbol{x}(t)=\boldsymbol{x}_{0}+$ $c \boldsymbol{\mu}\left(t-t_{0}\right)$

$$
\begin{aligned}
\frac{\mathrm{d}}{\mathrm{d} t} A^{i}(t, \boldsymbol{x}(t)) & =\frac{\partial}{\partial t} A^{i}(t, \boldsymbol{x}(t))+\frac{\partial}{\partial x^{j}} A^{i}(t, \boldsymbol{x}(t)) c \mu^{j} \\
& =\frac{\alpha_{*}}{\beta_{*}} \frac{\partial}{\partial t^{*}} A^{i}\left(t^{*}, \boldsymbol{x}(t)\right)+\frac{\partial}{\partial x^{j}} A^{i}\left(t^{*}, \boldsymbol{x}(t)\right) c \mu^{j}
\end{aligned}
$$


one can prove that a time derivative of (C.33) exactly coincides with the post-Minkowskian equations of motion for a photon (C.11)-(C.22) provided that $\boldsymbol{v}$ is taken to be equal to $\boldsymbol{\mu}$ in the latter (this is allowed within the first post-Minkowskian approximation as discussed above).

A different way to derive the Kopeikin-Schäfer solution (C.28)-(C.33) for the case of bodies moving with constant velocities is given by Klioner (2003b) who has shown how to derive this solution combining the post-Newtonian solution (B.15)-(B.26) for a motionless body $\left(\boldsymbol{v}_{\mathrm{A}}=0\right)$ with a suitable Lorentz transformation.

\section{Appendix D: Two point boundary value problem for the analytical solutions}

Let us consider analytical equations of light propagation in the form valid for both analytical models discussed above

$$
\begin{aligned}
\boldsymbol{x}(t)= & \boldsymbol{x}\left(t_{0}\right)+c \boldsymbol{\mu} s\left(t_{0}\right)\left(t-t_{0}\right)+\Delta \boldsymbol{x}\left(t_{0}, t\right) \\
& -\Delta \dot{\boldsymbol{x}}\left(t_{0}\right)\left(t-t_{0}\right)+O\left(\epsilon^{2}\right), \\
\frac{1}{c} \dot{\boldsymbol{x}}(t)= & \boldsymbol{\mu} s\left(t_{0}\right)+\frac{1}{c} \Delta \dot{\boldsymbol{x}}(t)-\frac{1}{c} \Delta \dot{\boldsymbol{x}}\left(t_{0}\right)+O\left(\epsilon^{2}\right),
\end{aligned}
$$

with

$\Delta \boldsymbol{x}\left(t_{0}, t_{0}\right)=0$,

$\lim _{t \rightarrow-\infty} \frac{1}{c} \Delta \dot{\boldsymbol{x}}(t)=0$.

These two conditions can be proven to be valid for both analytical models considered above. The small parameter $\epsilon$ can be identified with $G$ for the post-Minkowskian solution and with $c^{-2}$ for the post-Newtonian solution, so that in both solutions $\Delta \boldsymbol{x}\left(t_{0}, t\right)=O(\epsilon)$ and $c^{-1} \Delta \dot{\boldsymbol{x}}(t)=O(\epsilon)$.

Let us define several vectors

$\sigma=\lim _{t \rightarrow-\infty} \frac{1}{c} \dot{x}(t)$

$\boldsymbol{n}=\boldsymbol{n}(t)=\frac{\dot{\boldsymbol{x}}(t)}{|\dot{\boldsymbol{x}}(t)|}$,

$\boldsymbol{k}=\boldsymbol{k}\left(t_{0}, t\right)=\frac{\boldsymbol{R}\left(t_{0}, t\right)}{\left|\boldsymbol{R}\left(t_{0}, t\right)\right|}$,

$\boldsymbol{R}=\boldsymbol{R}\left(t_{0}, t\right)=\boldsymbol{x}(t)-\boldsymbol{x}\left(t_{0}\right)$.

These definitions are illustrated in Fig. 2. Vector $\sigma$ is the unit direction of the light path at past null infinity $(t \rightarrow-\infty)$. Vector $\boldsymbol{n}(t)$ is the unit direction of light propagation at time moment $t$ (so that $\boldsymbol{n}\left(t_{0}\right)=\boldsymbol{\mu}$ and $\lim _{t \rightarrow-\infty} \boldsymbol{n}(t)=\boldsymbol{\sigma}$ ). Vector $\boldsymbol{k}$ is the unit vector connecting the points $\boldsymbol{x}\left(t_{0}\right)$ and $\boldsymbol{x}(t)$. Now, if one considers the points $\boldsymbol{x}\left(t_{0}\right)$ and $\boldsymbol{x}(t)$ as given constants, one gets from (D.1)-(D.4) the following formulas approximately solving the two point boundary value problem for the differential equations of motion

$\boldsymbol{\sigma}=\boldsymbol{\mu}-\boldsymbol{\mu} \times\left(\frac{1}{c} \Delta \dot{\boldsymbol{x}}\left(t_{0}\right) \times \boldsymbol{\mu}\right)+O\left(\epsilon^{2}\right)$,

$s(t)=1+\frac{1}{c} \boldsymbol{\mu} \cdot \Delta \dot{\boldsymbol{x}}(t)+O\left(\epsilon^{2}\right)$,

$$
\begin{aligned}
\boldsymbol{n}=\boldsymbol{\mu} & +\boldsymbol{\mu} \times\left(\left[\frac{1}{c} \Delta \dot{\boldsymbol{x}}(t)-\frac{1}{c} \Delta \dot{\boldsymbol{x}}\left(t_{0}\right)\right] \times \boldsymbol{\mu}\right)+O\left(\epsilon^{2}\right), \\
\boldsymbol{k}= & \boldsymbol{\mu}+\boldsymbol{\mu} \times\left(\left[-\frac{1}{c} \Delta \dot{\boldsymbol{x}}\left(t_{0}\right)+\frac{1}{|\boldsymbol{R}|} \Delta \boldsymbol{x}\left(t_{0}, t\right)\right] \times \boldsymbol{\mu}\right) \\
& +O\left(\epsilon^{2}\right) \\
\boldsymbol{n}= & \boldsymbol{k}+\boldsymbol{k} \times\left(\left[\frac{1}{c} \Delta \dot{\boldsymbol{x}}(t)-\frac{1}{|\boldsymbol{R}|} \Delta \boldsymbol{x}\left(t_{0}, t\right)\right] \times \boldsymbol{k}\right) \\
& +O\left(\epsilon^{2}\right) .
\end{aligned}
$$

Note that (D.9) follows from (D.11) for $t \rightarrow-\infty$, that (D.13) is a combination of (D.11) and (D.12), and that (D.10) agrees with the expressions (B.14) and (C.26) for $s(t)$ given above for the post-Newtonian and the post-Minkowskian solutions, respectively. From (D.12) and the corresponding expressions for $\dot{\boldsymbol{x}}(t)$ and $\Delta \boldsymbol{x}\left(t_{0}, t\right)$ one can see that for a given impact parameter of the light ray the difference between vectors $\boldsymbol{k}$ and $\boldsymbol{\mu}$ becomes smaller for greater distances between the gravitating body and point of emission $\boldsymbol{x}\left(t_{0}\right)$.

Note that $\Delta \boldsymbol{x}\left(t_{0}, t\right), \Delta \dot{\boldsymbol{x}}(t)$ and $\Delta \dot{\boldsymbol{x}}\left(t_{0}\right)$ on the right-hand sides of (D.9)-(D.13) depend on $\boldsymbol{\mu}$. Formally, considering only analytical orders of magnitude one could replace $\boldsymbol{\mu}$ by $\boldsymbol{k}$ in these formulas. However, this works well only when the impact parameters computing for the unperturbed trajectories with directions $\boldsymbol{\mu}$ and $\boldsymbol{k}$ are sufficiently close to one another. This is not always the case. For example, this assumption is wrong in the gravitational lens limit. It is more reliable to calculate vector $\boldsymbol{\mu}$ from given $\boldsymbol{k}$ by a numerical inversion of (D.12) and then calculate $\boldsymbol{n}$ from (D.11).

\section{References}

Bienaymé, O., \& Turon, C. 2002, (ed.) GAIA: A European Space Project (Les Ulis: EDP Sciences)

Brumberg, V. A. 1991, Essential Relativistic Celestial Mechanics (Bristol: Hilger)

Brumberg, V. A., Klioner, S. A., \& Kopejkin, S. M. 1990, in Inertial Coordinate System on the Sky, ed. J. H. Lieske, \& V. K. Abalakin (Dordrecht: Kluwer), 229

Char, B. W., Geddes, K. O., Gonnet, G. H., et al. 1993, MAPLE V Library Reference Manual (New York: Springer)

de Felice, F., Bucciarelli, B., Lattanzi, M. G., \& Vecchiato, A. 2001, A\&A, 373, 336

de Felice, F., Lattanzi, M. G., Vecchiato, A., \& Bernacca, P. L. 1998, A\&A, 332, 1133

de Felice, F., Vecchiato, A., Bucciarelli, B., Lattanzi, M. G., \& Crosta, M. 2000, in Towards Models and Constants for SubMicroarcsecond Astrometry, ed. K. J. Johnston, D. D. McCarthy, B. J. Luzum, \& G. H. Kaplan (Washington: US Naval Observatory), 314

ESA. 2000 GAIA: Composition, Formation and Evolution of the Galaxy, Concept and Technology Study Report (ESA-SCI[2000]4) (Noordwijk: ESA)

Everhart, E. 1974, Celest. Mech., 10, 35

Everhart, E. 1985, in Dynamics of Comets: Their Origin and Evolution, ed. A. Carusi, \& G. B. Valsecci, Astrophysics and Space Science Library 115 (Dordrecht: Reidel), 185

Hellings, R. W. 1986, AJ, 91, 650 
IAU 2001, Information Bulletin, 88 (errata in IAU Information Bulletin, 89)

Jackson, J. D. 1974, Classical Electrodynamics (New York: Wiley)

Klioner, S. A. 1989, Soobschch. Inst. Prik. Astron. No. 6 (Communications of the Institute of Applied Astronomy, No. 6, St. Petersburg, in Russian)

Klioner, S. A. 1991, in Geodetic VLBI: Monitoring Global Change, ed. W. E. Carter, NOAA Technical Report NOS 137 NGS 49, 188 (Washington D.C.: American Geophysical Union)

Klioner, S. A. 2003a, AJ, 125, 1580

Klioner, S. A. 2003b, A\&A, 404, 783

Klioner, S. A., \& Kopeikin, S. M. 1992, AJ, 104, 897

Kopeikin, S. M. 1997, J. Math. Phys., 38, 2587

Kopeikin, S. M., \& Schäfer, G. 1999, Phys. Rev. D, 60, No. 124002
Kopeikin, S. M., \& Mashhoon, B. 2002, Phys. Rev. D, 65, No. 64025 Mignard, F. 2002, Considerations on the orbit of GAIA for simulations, GAIA-FM-011, manuscript available from the GAIA Livelink archive

Perryman, M. A. C., et al. 2001, A\&A, 369, 339

Shao, M. 1998, Proc. SPIE, 3350, 536

Soffel, M., Klioner, S. A., Petit, G., et al. 2003, The IAU 2000 resolutions for astrometry, celestial mechanics and metrology in the relativistic framework: explanatory supplement, AJ, accepted

Weissman, P. R., McFadden, L.-A., \& Johnson, T. V. (eds.) 1999, Encyclopedia of the Solar System (San Diego: Academic Press)

Will, C. M. 1993, Theory and experiment in gravitational physics (Cambridge: Cambridge University Press) 